\title{
INFLUÊNCIA DO FLUXO ARTERIAL PERIFÉRICO NA CAPACIDADE FUNCIONAL EM INDIVÍDUOS HIPERTENSOS
}

\author{
Elisa Kronbauer Pereira ${ }^{1}$ \\ Bárbara da Costa Flores ${ }^{2}$ \\ Cássia da Luz Goulart ${ }^{3}$ \\ Juliano Rodrigues Adolfo ${ }^{4}$ \\ Dulciane Nunes Paiva ${ }^{5}$ \\ Dannuey Machado Cardoso 6
}

\begin{abstract}
RESUMO
A Hipertensão Arterial Sistêmica (HAS) é caracterizada por disfunção endotelial, ocorrendo ainda aumento da resistência vascular periférica e hipertrofia/hiperplasia da parede vascular. Estes fatores podem contribuir para o surgimento da Doença Arterial Periférica (DAP) e esta, consequentemente, para a redução da capacidade funcional. Sendo assim, o presente estudo objetivou avaliar a influência do fluxo arterial periférico em membros inferiores sobre a capacidade funcional em indivíduos com HAS. Tratou-se de estudo transversal que avaliou 16 indivíduos hipertensos (6 homens, idade 60,4 $\pm 8,7$ anos). Foi obtido o Índice Tornozelo-Braquial (ITB) antes e após o Teste do Degrau de 4 minutos (TD4M) pela medida da pressão arterial sistólica braquial e em tornozelos, sendo os indivíduos classificados em Grupo ITB Normal (GITBN) e Grupo DAP (GDAP). 0 GDAP apresentou ITB pré-TD4M significativamente menor que 0 GITBN ( $p<0,001)$. Houve reducãa significativa do ITB pré para o pós-TD4M apenas no GDAP ( $p=0,029)$. Foi observada correlação moderada entre o número de degraus obtidos no TD4M e 0 ITB pré-TD4M na amostra total ( $r=0,700, p=0,003)$, tendo o ITB pré-TD4M influenciado no desempenho do teste $\left(R^{2} \quad=0,221\right)$. A limitação da capacidade funcional em pacientes com DAP está atribuída à diminuição do fluxo sanguíneo induzido por obstrușãăo. Em nossa amostra, o TD4M demonstrou ser um teste válido para avaliar indivíduos com HAS e ITB indicativo de $\operatorname{DAP}(<0,90)$, visto que houve associação entre o número de degraus e o ITB avaliado antes do teste do degrau.
\end{abstract}

Palavras-chave: Índice Tornozelo-Braço. Teste de esforço. Hipertensão.

\section{INFLUENCE OF PERIPHERAL BLOOD FLOW IN FUNCTIONAL CAPACITY IN HYPERTENSIVE INDIVIDUALS}

\begin{abstract}
Systemic arterial hypertension (SAH) is characterized by endothelial dysfunction, although there was an increase in peripheral vascular resistance and hypertrophy/hyperplasia of the vascular wall. These factors may contribute to the onset of peripheral arterial disease (PAD) and this consecutively to reduced functional capacity. Therefore, this study aimed to evaluate the influence of peripheral blood flow in the lower limbs on functional capacity in patients with SAH. This was cross-sectional study that evaluated 16 hypertensive patients (6 men, age 60.4 \pm 8.7 years). The ankle-brachial index (ABI) was obtained before and after the 4 minutes Step Test (4MST) by measuring brachial systolic blood pressure and ankles, and the individuals classified in Normal ITB Group (NITBG) and PAD Group (PADG). The GDAP presented ABI pre-4MST significantly smaller than the NITBG $(p<0.001)$. There was a significant reduction in pre ABI for post-4MST only PADG $(p=0.029)$. It observed moderate correlation between the number of steps obtained in 4MST and ABI pre-4MST the total sample $(r=0.700, p=0.003)$, and the $A B I$ pre-4MST influenced the test results $\left(R_{2 a \text { instado }}=0.221\right)$. The limitation of functional capacity in patients with PAD is attributed to decreased blood flow induced by obstruction. In our sample, the 4MST proved to be a valid test to evaluate patients with SAH and ABI indicative of PAD $(<0.90)$, whereas there was an association between the number of steps and the $A B I$ evaluated before the step test.
\end{abstract}

Keywords: Ankle Brachial-Index. Exercise test. Hypertension.

\footnotetext{
1 Fisioterapeuta - Universidade de Santa Cruz do SUL/ Unisc elisakronp@gmail.com

2 Acadêmica de Fisioterapia - Universidade de Santa Cruz do SUL/ Unisc. babiflores94@hotmail.com

3 Acadêmica de Fisioterapia - Universidade de Santa Cruz do SUL/ Unisc. luz.cassia@hotmail.com

${ }^{4}$ Residente do Programa de Pós-Graduação em Residência Multiprofissional Integrada em Saúde - Hospital Santa Cruz (HSC)/Unisc. Bacharel e Licenciado em Educação Física, Pontifícia Universidade Católica do Rio Grande do Sul (PUCRS). juliano_rodolfo@hotmail.com

5 Doutora em Ciência Médicas pela Universidade Federal do Rio Grande do Sul (UFRGS), Docente Programa de Pós-Graduação em Promoção da Saúde. dulciane@unisc.br

6 Mestre e doutorando do Programa de Pós-Graduação em Pneumologia da UFRGS. dannueyc@unisc.br
} 
A Hipertensão Arterial Sistêmica (HAS) é uma condição caracterizada pela disfunção endotelial, que tem importância fundamental na sua gênese e manutenção, sendo acompanhada por mudanças estruturais e funcionais do sistema vascular em resposta às variações hemodinâmicas (Regenga, 2012). Neste aspecto, os parâmetros fisiológicos cardíaco e arterial podem ser medidos por testes não invasivos, bem como podem ser utilizados para a identificação de disfunções cardiovasculares (Paiva et al., 2015).

O Índice Tornozelo-Braquial (ITB) é um método utilizado para avaliar o fluxo arterial periférico e para triagem da Doença Arterial Periférica (DAP) (Kim; Wattanakit; Gornik, 2012). Descrito por Winsor em 1950, este índice foi primeiramente proposto para o diagnóstico não invasivo. Posteriormente o ITB tornou-se um indicador de aterosclerose e um marcador de prognóstico para eventos cardiovasculares e comprometimento funcional, mesmo na ausência de sintomas (Aboyans et al., 2012). A Capacidade Funcional (CF), por sua vez, é de extrema importância, pois pode determinar a tolerância ao esforço físico, orientar a prescrição de exercício e avaliar a necessidade e a resposta a determinados tipos de intervenções terapêuticas (Regenga, 2012).

O Teste do Degrau de 4 Minutos (TD4M) é influenciado pela função pulmonar e musculatura periférica do indivíduo, sendo de boa reprodutibilidade e aplicação clínica. Conjectura-se que uma redução no fluxo arterial periférico poderia interferir no desempenho do TD4M. Dessa forma, o objetivo deste estudo foi avaliar a influência do fluxo arterial periférico sobre a capacidade funcional de indivíduos hipertensos.

\section{MÉTODO}

Trata-se de um estudo transversal, que avaliou portadores de hipertensão arterial, selecionados a partir de amostra de conveniência em grupos de hipertensos na Estratégia de Saúde da Família no Bairro Menino Deus e Bairro Glória/Imigrante, na cidade de Santa Cruz do Sul - RS. Foram avaliados indivíduos de ambos os sexos, com diagnóstico clínico de HAS leve a moderada, segundo a VI Diretriz Brasileira de Hipertensão (Nobre, 2010), idade entre 50 e 70 anos, sedentários (menos de $30 \mathrm{~min}$ de exercício físico três vezes por semana), tendo sido incluídos aqueles com função pulmonar normal, hemodinamicamente estáveis e que assinaram o Termo de Consentimento Livre e Esclarecido. Foram excluídos aqueles com HAS grave, comprometimento osteomuscular como artrose de quadril e/ou joelho, com sequelas neurológicas e alterações de equilíbrio que impossibilitassem ou comprometessem a realização do TD4M ou a medida da pressão arterial braquial. O presente projeto foi aprovado pelo Comitê de Ética em Pesquisa da Universidade de Santa Cruz do Sul, sob protocolo número 639.378 .

\section{Procedimento}

Inicialmente foram coletadas as variáveis antropométricas (sexo, idade, peso, altura e IMC) e após, aferidos os sinais vitais e avaliado o ITB. Na sequência foi realizada a espirometria para avaliação da função pulmonar e avaliada a percepção de esforço por meio da Escala de Borg. Na sequência foi realizado o TD4M, quando ao final do teste os sinais vitais, a percepção de esforço e o ITB foram novamente aferidos.

\section{Avaliação dos sinais vitais}

Para avaliação da frequência respiratória (FR) foi contado o número de incursões respiratórias em um minuto. Já para a avaliação da frequência cardíaca (FC) foi utilizado um cardiofrequencímetro (Polar, IR Interface ${ }^{\mathrm{TM}}$, EUA) e para a saturação periférica de oxigênio $\left(\mathrm{SpO}_{2}\right)$ um oxímetro portátil (Zondan, A4, China). A Pressão Arterial Sistólica (PAS) e Diastólica (PAD) foram aferidas com o paciente em sedestação, em ambos os membros superiores, pelo método palpatório e adotado o membro superior com maior valor, conforme recomendação da VI Diretriz Brasileira de Hipertensão (Nobre, 2010), e para tal utilizado um esfigmomanômetro anaeroide (Welchallyn Durashock DS44, Tycos) avaliado quanto à fidedignidade no Instituto Nacional de Metrologia, Qualidade e Tecnologia (Inmetro) e um estetoscópio (Littman classic II). 


\section{Fluxo arterial periférico}

O fluxo arterial periférico foi avaliado por meio do ITB, realizadas duas medidas por paciente (pré e pós TD4M), com tal variável sendo obtida com a medida da Pressão Arterial Sistólica braquial em ambos os membros superiores e ambos os tornozelos ao nível da artéria pediosa e tibial posterior, conforme recomendação da American Heart Association (Aboyans et al., 2012). Para assegurar a correta ausculta do pulso utilizou-se Doppler vascular portátil (MEDPEJ, DV - 2001, Ribeirão Preto, Brasil). Para o cálculo do ITB foi validada a maior medida da PAS braquial e em tornozelo. $\mathrm{O}$ indivíduo foi classificado a partir do resultado do índice, considerando que valores de ITB abaixo de 0,9 indicam presença de doença oclusiva significativa e valores acima de 1 indicam normalidade (Kim; Wattanakit; Gornik, 2012). O ITB foi obtido antes e imediatamente após a realização do TD4M.

\section{Função pulmonar}

A avaliação dos volumes pulmonares foi realizada através de espirometria (EasyOne ${ }^{\circledR}$, Modl 2001, Diagnostic Spirometer, Suíça), devidamente calibrado (GlobalMed Suporte de Materiais Terapêuticos Ltda., novembro/2014). A manobra expiratória forçada foi efetuada com os indivíduos em sedestação, sendo repetida três vezes, no mínimo, até que a curva expiratória apresentasse um traçado satisfatório (Miller et al., 2005). Foram avaliadas a Capacidade Vital Forçada (CVF), Volume Expiratório Forçado no Primeiro Segundo $\left(\mathrm{VEF}_{1}\right)$, relação $\mathrm{VEF}_{1} / \mathrm{CVF}$ e o Pico do Fluxo Expiratório (PFE). Foram validadas as curvas que apresentaram o melhor desempenho de acordo com os valores preditos (Pereira et al., 1992).

\section{Capacidade funcional}

A capacidade funcional foi avaliada por meio do TD4M, sendo este realizado com monitorização prévia da $\mathrm{PAS}, \mathrm{PAD}, \mathrm{FC}, \mathrm{SpO}_{2}$ e percepção de esforço mediante a escala de Borg (Borg, 1982). O teste foi feito com a utilização de um degrau de 20 $\mathrm{cm}$, sem apoio para membros superiores, os quais permaneceram estacionários ao longo do corpo. Os princípios gerais do teste foram baseados na atual recomendação da ATS, e todos os pacientes realizaram treinamentos prévios (AMERICAN THORACIC SOCIETY, 2002). Os voluntários foram instruídos a subir e descer da plataforma o mais rápido possível, em cadência livre e no seu próprio ritmo, durante um período de quatro minutos. Também foram informados de que poderiam diminuir o ritmo, se necessário, e até mesmo fazer paradas para descanso. A cada minuto foram dadas frases de encorajamento, usando um mesmo tom de voz. Os voluntários também foram informados a cada minuto quanto tempo de que ainda dispunha para subir o degrau. Ao final, os mesmo sinais vitais e a percepção do esforço obtida no pré-teste foram novamente avaliados, assim como registrado o número de degraus atingidos.

\section{ANÁLISE DOS DADOS}

O cálculo amostral foi baseado no piloto do estudo com cinco pacientes, e para detectar uma associação significativa entre o número de degraus atingidos no TD4M e o escore obtido no ITB, com um nível de significância de 5\% (bicaudal), com poder de $80 \%$ e acrescida uma perda amostral de $10 \%$, seria necessário um tamanho de amostra de 15 pacientes.

Os dados foram analisados por meio do software estatístico SPSS (versão 20.0, EUA). O teste não paramétrico de Shapiro-Wilk foi utilizado para verificar a normalidade da distribuição dos dados que foram apresentados em média e desvio padrão. Para a comparação entre o ITB, sinais vitais e percepção de esforço no pré e pós foi empregado o teste $t$ de Student, assim como para comparação entre a diferença dos valores pré e pós entre os grupos. Para avaliar a associação entre o número de degraus alcançados no TD4M e o ITB foi utilizada a correlação de Pearson. O modelo de regressão linear múltipla foi utilizado para avaliar o efeito das va- 
riáveis independentes sobre o número de degraus atingidos no TD4M. A significância do modelo final foi avaliada pelo teste $\mathrm{F}$ da Anova e a qualidade do ajuste pelo coeficiente de determinação ajustado $\left(\mathrm{R}^{2}\right.$ ajustado). Os resíduos foram avaliados segundo as suposições de normalidade, variância constante e independência. Para efeito de significância estatística foi adotado um $\mathrm{p}<0,05$.

\section{RESULTADOS}

Foram avaliados 26 indivíduos; 7 deles foram excluídos por não completarem o programa de avaliações, 2 por terem apresentado problema osteomuscular e outro por estar com pico hipertensivo no momento da avaliação. A amostra final, então, foi composta por 16 sujeitos, os quais foram alocados em Grupo ITB Normal (GITBN, $n=10)$ e Grupo DAP (GDAP, $n=6$ ) de acordo com o resultado do ITB pré-TD4M. As características antropométricas, função pulmonar e as medicações utilizadas são apresentadas na Tabela 1, em que não foi observada diferença significativa entre os grupos. Quanto ao uso de medicações, a classe de betabloqueadores utilizada foi o propranolol em ambos os grupos, não havendo uso de anticoagulantes orais. Não foi observado o uso habitual de álcool pelos pacientes de ambos os grupos.

Tabela 1 - Características dos grupos avaliados

\begin{tabular}{lccc}
\hline \multicolumn{1}{c}{ Variáveis } & $\begin{array}{c}\text { Grupo ITB } \\
\text { Normal }\end{array}$ & $\begin{array}{c}\text { Grupo } \\
\text { DAP }\end{array}$ & *p-valor \\
& $(\mathrm{n}=10)$ & $(\mathrm{n}=6)$ & \\
\hline Sexo masculino, n (\%) & $4(40,0)$ & $2(33,3)$ & 0,608 \\
Idade (anos) & $61,4 \pm 9,04$ & $59,5 \pm 8,4$ & 0,684 \\
Altura (cm) & $162,8 \pm 7,9$ & $161,8 \pm 8,1$ & 0,818 \\
Peso (Kg) & $71,4 \pm 10,8$ & $78 \pm 13,6$ & 0,306 \\
IMC (Kg/m $\left.{ }^{2}\right)$ & $25,7 \pm 3,9$ & $29,9 \pm 4,6$ & 0,079 \\
Função Pulmonar & & & \\
CVF (\%pred) & $71,4 \pm 22,2$ & $75,1 \pm 15,3$ & 0,721 \\
VEF $(\%$ pred) & $67,3 \pm 27,05$ & $79,8 \pm 13,9$ & 0,773
\end{tabular}

\begin{tabular}{|c|c|c|c|}
\hline $\mathrm{VEF}_{1} / \mathrm{CVF}$ (\%pred) & $91,4 \pm 15,07$ & $94,8 \pm 7,5$ & 0,614 \\
\hline PEF (\%pred) & $54,2 \pm 30,5$ & $49 \pm 7,9$ & 0,693 \\
\hline Tabagismo ativo, n (\%) & $2(20,0)$ & $2(33,3)$ & 0,489 \\
\hline $\begin{array}{l}\text { Tabagismo prévio, } n \\
(\%)\end{array}$ & $3(30,0)$ & $2(33,3)$ & 0,654 \\
\hline HAS, n $(\%)$ & $6(66,0)$ & $4(66,7)$ & 0,608 \\
\hline \multicolumn{4}{|l|}{ Medicações } \\
\hline $\begin{array}{l}\text { Betabloqueador, } \mathrm{n} \\
(\%)\end{array}$ & $2(20,0)$ & $1(16,7)$ & 0,696 \\
\hline IECA, n (\%) & $6(60,0)$ & $4(73,3)$ & 0,511 \\
\hline \multicolumn{4}{|l|}{ Diuréticos } \\
\hline Tiazídicos, n (\%) & $4(40,0)$ & $1(16,7)$ & 0,330 \\
\hline De alça, n (\%) & $2(20,0)$ & $0(0,0)$ & 0,424 \\
\hline Estatinas, n (\%) & $8(80,0)$ & $2(33,3)$ & 0,092 \\
\hline $\begin{array}{l}\text { Antiagregante pla- } \\
\text { quetário, } \mathrm{n}(\%)\end{array}$ & $3(30,0)$ & $1(16,7)$ & 0,473 \\
\hline $\mathrm{ACC}, \mathrm{n}(\%)$ & $4(40,0)$ & $1(16,7)$ & 0,330 \\
\hline BRA, n (\%) & $2(20,0)$ & $0(0,0)$ & 0,424 \\
\hline
\end{tabular}

ITB: Índice Tornozelo-Braquial; DAP: Doença Arterial Periférica; IMC: Índice de Massa Corporal; CVF: Capacidade Vital Forçada; $\mathrm{VEF}_{1}$ : Volume Expiratório Forçado no Primeiro Segundo; PEF: Pico Defluxo Expiratório; HAS: Hipertensão Arterial: Ieca: Inibidor da Enzima Conversora da Angiotensina; ACC: Antagonistas dos Canais de Cálcio; BRA: Bloqueadores dos Receptores da Angiotensina. Significância: $\mathrm{p}<0,05$.

Fonte: Dados dos Autores.

Quanto ao ITB obtido antes e após a realização do TD4M, o GDAP apresentou redução significativa em relação ao GITBN, no entanto, a variação do índice após o TD4M não se diferenciou entre os grupos (Figura 1). 
Figura 1 - Comportamento do ITB entre o grupo com Índice Tornozelo-Braquial (ITB) normal e grupo com Doença Arterial Periférica (DAP). Significância: $p<0,05$

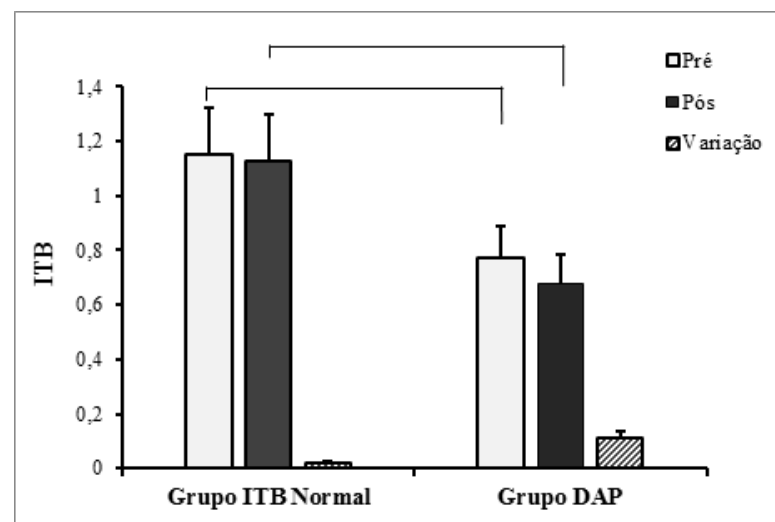

Fonte: Dados dos Autores.

Quanto ao número de degraus alcançados durante o teste, o GDAP apresentou número significativamente menor que o GITBN (Figura 2).

Figura 2 - Comparação da capacidade funcional, avaliada pelo Teste do Degrau de 4 Minutos (TD4) entre o grupo com Índice Tornozelo-Braquial (ITB) normal e grupo com Doença Arterial Periférica (DAP). Significância: $p<0,05$

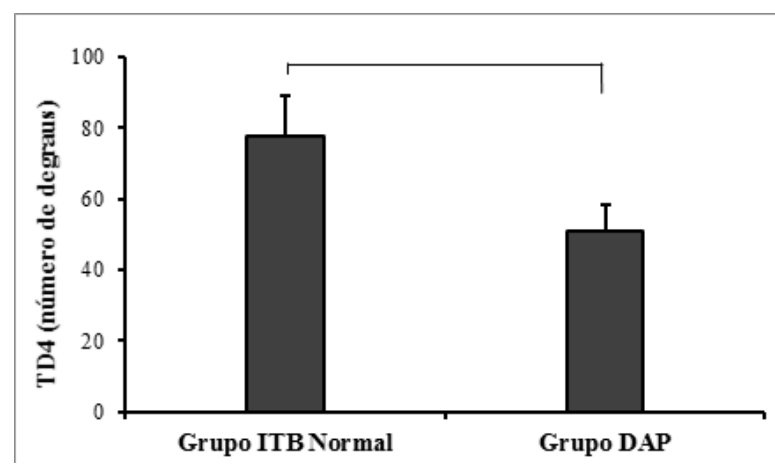

Fonte: Dados dos Autores.

Ao observarmos, no entanto, os sinais vitais obtidos pré e pós-teste, não houve alteração significativa dentro dos grupos avaliados, nem mesmo quando comparadas as variações do pré para o pós entre os grupos (Tabela 2). Ressalta-se que seis pacientes no GITBN e quatro no GDAP apresentaram pressão arterial controlada no momento da inclusão no estudo (PAS $<140 \mathrm{mmHg}$ e $\mathrm{PAD}<90 \mathrm{mmHg}$ ), segundo critério da VI Diretriz Brasileira de Hipertensão.
Tabela 2 - Comportamento dos sinais vitais e percepção de esforço pré e pós-teste do degrau

\begin{tabular}{|l|c|c|c|c|c|c|c|}
\hline \multirow{2}{*}{ Variáveis } & \multicolumn{3}{|c|}{ Grupo ITB Normal } & \multicolumn{3}{c|}{ Grupo DAP } & \multirow{2}{*}{ p-valor $^{b}$} \\
\cline { 2 - 8 } & Pré & Pós & p-valor & Pré & Pós & p-valor & \\
\hline FC (bpm) & $75,0 \pm 11,4$ & $82,9 \pm 16,2$ & 0,290 & $85,1 \pm 13,6$ & $92,8 \pm 19,5$ & 0,131 & 0,970 \\
\hline FR (irpm) & $18,1 \pm 1,5$ & $22,2 \pm 2,8$ & 0,983 & $19,6 \pm 1,9$ & $22,1 \pm 3,0$ & 0,159 & 0,320 \\
\hline $\begin{array}{l}\text { PAS } \\
(\mathrm{mmHg})\end{array}$ & $129,0 \pm 15,2$ & $157 \pm 21,1$ & 0,232 & $131,0 \pm 13,2$ & $144,7 \pm 17,4$ & 0,728 & 0,114 \\
\hline $\begin{array}{l}\text { PAD } \\
(\mathrm{mmHg})\end{array}$ & $68,5 \pm 31,8$ & $106,5 \pm 31,2$ & 0,122 & $71,6 \pm 30,6$ & $85 \pm 5,4$ & 0,848 & 0,260 \\
\hline $\begin{array}{l}\text { BORG } \\
\text { (escore) }\end{array}$ & $0 \pm 0$ & $2,7 \pm 0,5$ & 0,059 & $0 \pm 0$ & $3,75 \pm 2,8$ & 0,066 & 0,518 \\
\hline
\end{tabular}

ITB: Índice Tornozelo-Braquial; DAP: Doença Arterial Periférica; FC: Frequência Cardíaca; FR: Frequência Respiratória; PAS: Pressão Arterial Sistólica; PAD: Pressão Arterial Diastólica. ${ }^{a}$ Comparação intragrupo. ${ }^{b}$ Comparação entre os grupos. Significância: $p<0,05$.

Fonte: Dados dos Autores.

Nossos resultados demonstraram ainda uma associação direta e moderada entre o número de degraus obtidos no TD4M e o escore do ITB pré $(\mathrm{r}=0,700 ; \mathrm{p}=0,003)$, na amostra total de sujeitos (Figura 3).

Figura 3 - Associação entre o Índice Tornozelo-Braquial (ITB) e a capacidade funcional avaliada pelo teste do degrau de 4 minutos (TD). Significân-

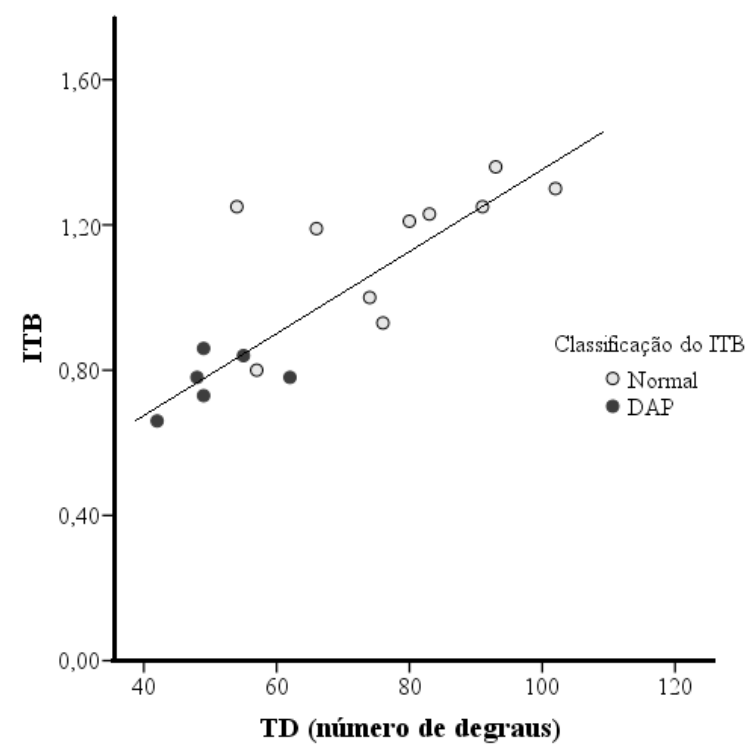

Fonte: Dados dos Autores. 
Após o ajuste do modelo de regressão linear a variável ITB pré foi independentemente associada com o número de degraus alcançados no TD4M na amostra estudada (Tabela 3).

Tabela 3 - Análise da regressão linear com o número de degraus atingidos no Teste do Degrau de 4 minutos e a variável independente

\begin{tabular}{|l|c|c|c|}
\hline Variável & Coeficiente B & IC 95\% & p-valor \\
\hline Constante & 32,841 & $8,79-66,4$ & 0,035 \\
\hline ITB & 32,412 & $2,08-62,7$ & 0,002 \\
\hline
\end{tabular}

Notas: ITB: Índice Tornozelo-Braquial; $\mathrm{R}_{\text {ajustado }}^{2}=0,221$; $\mathrm{F}=5,254(\mathrm{p}=0,030)$. IC: Intervalo de Confiança. Significância estatística: $p<0,05$. Fonte: Dados dos Autores

A variável independente contemplada neste modelo explicou aproximadamente $22 \%$ da variação no TD4M $\left(\mathrm{R}_{\text {ajustado }}^{2}=0,221\right)$.

\section{DISCUSSÃO}

Os mecanismos fisiopatológicos da hipertensão arterial como fator contribuinte para o desenvolvimento da doença vascular são complexos. Caracteriza-se, porém, como uma condição clínica multifatorial assinalada por coeficientes pressóricos elevados e sustentados, concomitante a alterações funcionais e/ou estruturais dos órgãos-alvo (coração, encéfalo, rins e vasos sanguíneos), além de alterações metabólicas, com consequente aumento do risco de eventos cardiovasculares fatais e não fatais (Lima; Barros; Oliveira, 2014). Ressalta-se que um fator importante para avaliação do ITB e correta caracterização da DAP é o momento em que este índice é avaliado, seja em repouso ou pós-exercício, pois com o exercício há um aumento do fluxo através de uma estenose fixa que leva a uma queda significativa na pressão tornozelo (Kim; Wattanakit; Gornik, 2012). Dessa forma, Sato et al. (2011) avaliaram em 35 sujeitos as alterações no ITB pós-exercício e a disfunção endotelial, sendo avaliada pela medida da vasodilatação mediada pelo fluxo (FMD) na artéria braquial, em idosos. Neste estudo não houve correlação entre a FMD e o ITB em repouso, entretanto ocorreu uma fraca correlação entre a FMD e o ITB pós-exercício. Sendo assim, os autores concluíram que o ITB pós-exercício parece ser um marcador alternativo e simples para a avaliação da função endotelial nos idosos. É importante mencionar, no entanto, que em nossa amostra nenhum sujeito apresentou ITB limítrofe ou alteração da classificação após o TD4M, sendo por este motivo adotados os valores pré-TD4M para classificação, observando-se que uma queda no ITB $\leq 0,9$ é indicativo de obstrução arterial hemodinamicamente significativa (Conte et al., 2015).

Apesar de termos utilizado o TD4M em paciente com alteração do fluxo arterial periférico, normalmente este é realizado em cardiopatas ou portadores de doenças respiratórias, mostrando-se útil na avaliação da tolerância ao exercício, visto que impõe alta demanda metabólica quando comparado ao TC6M. Além disso, é uma modalidade de baixo custo para predizer a aptidão cardiorrespiratória (Albuquerque et al., 2014).

É importante mencionar, contudo, que outros fatores também limitam o desempenho dos indivíduos, dentre eles a intolerância ao exercício, acompanhada por dispneia e fadiga muscular de membros inferiores (Andrade et al., 2012; Mazzocchi et al., 2012).

A limitação da capacidade funcional em paciente com DAP tem sido atribuída à diminuição do fluxo sanguíneo induzido por obstrução arterial aterosclerótica, no entanto a associação entre a gravidade da obstrução arterial e o estado funcional em paciente com DAP ainda permanece sem resposta (Hamburg; Balady, 2011). Alguns estudos têm demonstrado uma associação moderada entre o ITB e uma curta distância percorrida no TC6M entre os pacientes (Isquierdo-Porrera et al., 2005; Long et al., 2004; Silva et al., 2012), enquanto outra pesquisa relatou nenhuma associação do ITB com medidas funcionais (Szuba et al., 2006), comprovando que a hipertensão arterial e o tabagismo repercutem negativamente no TC6, evidenciando por meio de 
estudos que participantes que apresentaram fatores de risco cardiovascular caminharam significativamente menos no TC6 quando comparados aos indivíduos sem fatores de risco (Dourado, 2011). Nesse sentido, um estudo multicêntrico brasileiro demonstrou que a DAP prejudica significativamente a capacidade funcional e a qualidade de vida do paciente. Assim, a avaliação da capacidade funcional em indivíduos com risco de doença cardiovascular é uma ferramenta importante, que reflete esforços integrados no que diz respeito à saúde dos sistemas pulmonares e cardiovasculares, circulação periférica e sistêmica, unidades neuromusculares e metabolismo muscular (Gomes et al., 2015). Apesar dos resultados significativos, é importante mencionar algumas limitações do estudo, como o fato de não termos avaliado a presença de claudicação intermitente e o número de degraus livres de claudicação no GDAP, constituindo-se esta avaliação importante para identificar o impacto direto da redução do fluxo periférico no desempenho no TD4M. Além disso, o nível de atividade física poderia ter sido avaliado a fim de observar se esta variável também interferiu no desempenho do TD4M.

A relevância deste estudo está na utilização do TD4M para avaliação da DAP, uma vez que este teste pode reproduzir parte da limitação imposta pelo baixo fluxo arterial periférico encontrado nos pacientes com DAP, além de ser um teste simples e de baixo custo, podendo facilmente ser realizado para avaliação do paciente em um programa de reabilitação cardiovascular.

\section{CONCLUSÃO}

Em conclusão, nosso estudo demonstrou que o TD4M, mesmo sendo um teste simples e ainda pouco referenciado na literatura, pode ser útil na avaliação da DAP. Constatamos que o GDAP obteve um número significativamente reduzido de degraus quando comparados ao grupo normal, apresentando ainda uma associação moderada desta variável com o Índice Tornozelo-Braquial.

\section{REFERÊNCIAS}

ABOYANS, V. et al. Measurement and interpretation of the ankle-brachial index: A scientific statement from the Americam Heart Association. Circulation Journal of the America Heart Association, Boston, v. 126, n. 24, p. 2.890-2.909, nov./ dez. 2012.

ALBUQUERQUE, I. et al. Respostas cardiorrespiratórias durante dois testes de exercícios submáximos em participantes de um programa de reabilitação cardíaca: Resultados preliminares. Revista Saúde, Santa Maria, v. 40, n. 1, p. 117-124, jan./ jul. 2014.

AMERICAN THORACIC SOCIETY/STATEMENT. Guidelines for the Six-Minute Walk Test. American Journal of Respiratory and Critical Care. Medicine, New York, v. 166, n. 1, p. 111-117, jul. 2002.

ANDRADE, C. H. S. et al. O uso do teste do degrau para avaliação da capacidade de exercício em pacientes com doenças pulmonares crônicas. Jornal Brasileiro de Pneumologia. São Paulo, v. 38, n. 1, p. 116-124, abr./jul. 2012.

BORG, G. A. V. Psychophysical bases of perceived exertion. Medicine and Science in sports and exercice, Hagerstown, v. 14, n. 5, p. 377-381, 1982.

CONTE, M. S. et al. Society for Vascular Surgery pratice guidelines for atherosclerotic occlusive disease of the lower extremities: Management of asymptomatic diasease and claudication. Journal of Vascular Surgery, Chicago, v. 61, n. 5, p. 2-41, mar. 2015.

DOURADO, V. Z. Equações de referência para o teste de caminhada de seis minutos em indivíduos saudáveis. Arquivo Brasileiro de Cardiologia, São Paulo, v. 96, n. 6, p. 128-138, jun. 2011.

GOMES, T. et al. Association between the ankle-brachial index, intermittent claudication, and physical activity level: what is the influence on the functional capacity of patients with or at high risk of cardiovascular disease. International Journal of General Medicine, Reino Unido, v. 8, p. 55-62, jan. 2015. 
HAMBURG, N. M.; BALADY, G. J. Exercise rehabilitation in peripheral artery disease: Functional Impact and mechanisms of benefits. Circulation, 2011. DOI: 10,1161 / CIRCULATIONAHA.109.881888.

ISQUIERDO-PORRERA, A. M. et al. Relationship between objective measures of peripheral arterial disease severity to self-reported quality of life in older adults with intermittent claudication. Journal of Vascular Surgery, Chicago, v. 41, n. 4, p. 625630, abr. 2005.

KIM, E. H. WATTANAKIT, K. GORNIK, H. L. Using the ankle-brachial index to diagnose peripheral artery disease and assess cardiovascular risk. Cleveland Clinic Journal of Medicine, Lyndhurst, v. 79, n. 9, p. 651-661, set. 2012.

LIMA, E. R.; BARROS, A. R. C.; OLIVEIRA, C. A. N. Percepção dos clientes hipertensos acerca das complicações da hipertensão arterial sistêmica. Revista Interfaces, saúde, humanas e tecnologia, Juazeiro do Norte, v. 2, n. 5, set./nov. 2014.

LONG, J. et al. Correlation between ankle-brachial index, symptons, and healthrelated quality of life in patients with peripheral vascular disease. Journal of Vascular Surgery, Chicago, v. 39, n. 4, p. 723-727, abr. 2004.

MAZZOCCHI, C. S. et al. Comparação das variáveis fisiológicas no teste de caminhada de seis minutos e no teste da escada em portadores de doença pulmonar obstrutiva crônica. Revista Brasileira Medicina do Esporte, São Paulo, v. 18, n. 5, p. 296-299, 2012.

MILLER, M. R. et al. Standardisation of spirrometry. European Respiratory Journal, v. 26, n. 2, p. 319-338, ago. 2005.

NOBRE, F. V. I. Diretrizes Brasileiras de Hipertensão. Arquivo Brasileiro de Cardiologia, São Paulo, n. 1, p. 1-51, jul. 2010.

PAIVA, D. N. et al. Comportamento do índice tornozelo-braquial após exercício submáximo em portadores de doença pulmonar obstrutiva crônica. Fiep bulletin on-line, 2015. DOI: 10.16887/85. a1.42.
PEREIRA, C. A. C. et al. Valores de referência para a espirometria em uma amostra da população brasileira adulta. Jornal de Pneumologia, São Paulo, v. 18, n. 1, p. 10-22, 1992.

REGENGA, Marisa. Fisioterapia em cardiologia: da unidade de terapia intensiva à reabilitação. 2. ed. São Paulo: Roca. 2012.

SATO, S. et al. Post-exercise ankle-brachial pressure index demonstrates altered endothelial function in the elderly. Journal Japonese Clinical Medicine, Japão, v. 2, p. 21-24, jun. 2011.

SILVA, R. C. G. et al. Limitação funcional e claudicação intermitente: impacto das medidas de pressão arterial. Arquivo Brasileiro de Cardiologia, São Paulo, v. 98, n. 2, p. 161-166, fev. 2012.

SZUBA, A. et al. Limb hemodynamics are not predictive of functional capacity in patients with PAD. Vascular medicine, v. 11, n. 3, p. 155-163, nov. 2006.

Recebido em: 13/7/2015

Aceito em: 20/4/2016 